\title{
Pregnancy outcomes in living kidney donors
}

New findings suggest that the incidences of gestational hypertension and pre-eclampsia might be increased in kidney donors compared with nondonors. Researchers Amit Garg and colleagues-who presented these data at ASN Kidney Weekhighlight the need to inform women of childbearing age who are thinking about donating a kidney of the potential effects of nephrectomy on pregnancy risks.

"Whether kidney donation might affect the outcomes of future pregnancies is one of the most important questions that young women who are considering donation ask our transplant team," explains Garg. "However, the findings of two previous studies that reported increased risks of gestational hypertension and pre-eclampsia after kidney donation have been debated, and many transplant centres haven't incorporated this information into their informed-consent process."

To investigate the effect of donor nephrectomy on pregnancy outcomes, Garg and colleagues conducted a retrospective cohort study using data from four linked databases in Ontario, Canada. Their analysis included 85 women who donated a kidney between 1 July 1992 and 30 April 2009, and 510 matched nondonors with similar baseline indicators of general health. To be eligible for inclusion, participants must have experienced at least one pregnancy of $\geq 20$ weeks gestation during follow-up until 31 March 2013; 131 pregnancies were reported in the living donor group and 788 pregnancies in the nondonor group.

The researchers found that in their cohort, kidney donors were 2.4 times more likely to be diagnosed with gestational hypertension or pre-eclampsia than were nondonors, with an

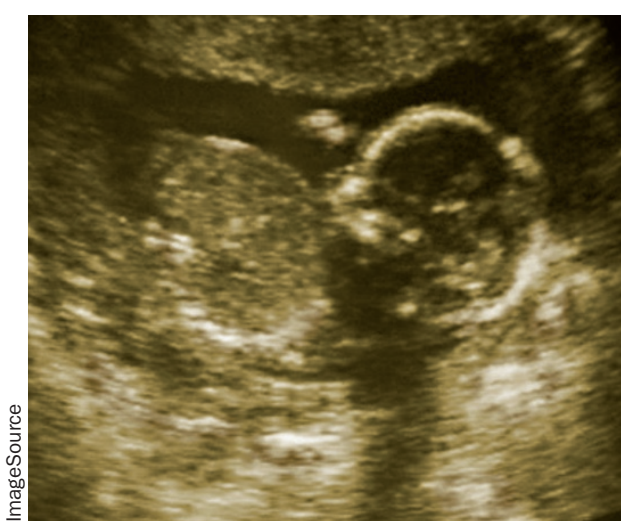

incidence of $11.5 \%$ in donor pregnancies compared with $4.8 \%$ in nondonor pregnancies. The increased risk of these complications in donors versus nondonors was significantly higher among those aged $>32$ years than in younger women. However, the incidences of caesarean section, postpartum haemorrhage, preterm birth ( $<37$ weeks) and low birth weight $(<2.5 \mathrm{~kg})$ did not differ significantly between donors and nondonors. No stillbirths, maternal or neonatal deaths were reported and most kidney donors did not experience pregnancy complications.

"We anticipate that most women of reproductive potential who are interested in donating a kidney to a loved one will find the risks described in this study acceptable and manageable, and will choose to proceed with donation," comments Garg. "We have shared the study results with past kidney donors and they were appreciative of the fact that we have obtained a more accurate estimate of the effects of donation on pregnancy. They all said that this new information would not have stopped them choosing to donate their kidney."

Ellen F. Carney

Original article Garg, A. X. et al. Gestational hypertension and preeclampsia in living kidney donors. N. Engl. J. Med. doi:10.1056/ NEJMoa1408932 